PREDICTION OF MACHINE TOOL SPINDLE'S DYNAMICS BASED ON A THERMO-MECHANICAL MODEL

P. Kolar, T. Holkup

Research Center for Manufacturing Technology, Faculty of Mechanical Engineering, CTU in Prague, Czech Republic

e-mail: p.kolar@rcmt.cvut.cz

This paper presents a thermo-mechanical model of spindles with rolling bearings. The heat generated in the bearings and the motor is transferred to the ambient air, the motor coolant and the spindle head structure. It warms the spindle up and causes thermal deformations of spindle parts. Thermal deformation affects significantly properties (internal loads, stiffness) of rolling bearings and need to be considered in predictive models of spindles' dynamics.

The structure of the transient model contains thermo-mechanical loops which update the model with respect to the instantaneous condition of the spindle. The changes of the bearings' internal condition and stiffness are calculated using the Jones' nonlinear theory. The thermo-mechanical spindle model predicts temperature distribution, thermal growth of spindle parts, and transient changes in bearing stiffness and contact loads under specified operating conditions. The predicted bearing properties are then used to estimate the variation of natural frequencies and dynamic stiffness of the spindle.

Keywords

Spindle, Rolling bearing, FEM modeling, Spindle dynamics, Thermal expansion

\footnotetext{
1. Introduction

The main task of computational models of machine tool spindles is prediction of their frequency response function (FRF) at the spindle nose or at the tool tip. The FRF is the key information for further optimization of cutting parameters and for estimation of cutting stability. For building an accurate model of a spindle system it is necessary to mathematically describe the following:

- mechanical parts (tool, tool holder, spindle shaft and housing);

- spindle bearings;

- interfaces (tooling interface, supporting structure - the machine tool);

- damping.
}

Stiffness of spindle bearings has significant influence on spindle dynamics. The models presented in [Cao 2004], [Altintas 2005], [Rantatalo 2007] use the bearing model developed by [Jones 1960] that describes properties of bearings with respect to their running conditions - speed and boundary conditions applied on bearing rings. However, the changes of bearing condition due to thermo-mechanical issues during spindle operation are neglected in these models. The paper presents a method which considers mechanical as well as thermal issues and it is demonstrated on a particular example of a grinding spindle.

\section{Structure of the model}

The presented thermo-mechanical model of spindles is a combination of the thermo-mechanical model [Holkup 2006] and a model of spindle dynamics [Kolar 2007]. The structure of the model is shown in Figure 1. Input data describe the spindle design and working conditions which are: spindle geometry, motor parameters, shaft revolu- tions, shaft load, bearing type, arrangement and preload. This information is the basis for building the two-dimensional thermo-mechanical model of the spindle (see chapter 3 ) which produces the output data - the relative displacement of bearing rings for particular conditions: speed, spindle power and time from the spindle's startup. The rings' displacement of each bearing is passed to the threedimensional mechanical model of the spindle (see chapter 4) which predicts the spindle dynamics. If more running conditions (e.g. different speed / power / time from the spindle's startup) need to be investigated, both models have to be updated.

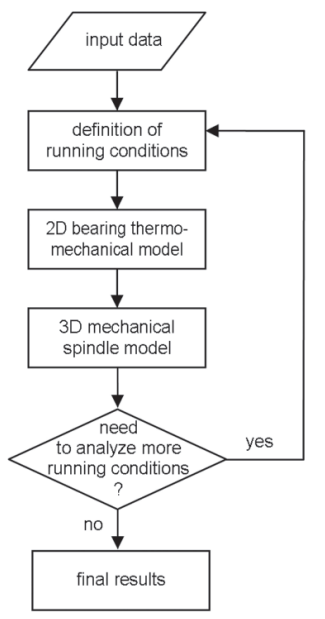

Figure 1. Structure of the thermo-mechanical model of spindles.

\section{Thermo-mechanical model of spindle rolling bearings}

Many problems of modern high-speed spindles are caused by thermal expansion of spindle parts. During operation, heat is produced by rolling bearings, electric motor and cutting process. Preloaded angular contact ball bearings are commonly used to obtain high stiffness of spindles and two main preload concepts can be distinguished:

- constant force preload using springs;

- position preload using rigid spacers.

In case of constant force preload - thermal expansion affects the relative position of bearing rings in radial direction and axial position adapts so that the preload remains constant. Then contact angles are affected and bearing properties change slightly.

In case of position preload, thermal expansion of stiff parts (shaft, housing, spacers, bearing rings, balls) can cause rapid changes in preload which affect the amount of heat produced in bearings. This closed-loop effect cause significant changes in bearing condition and properties which can lead to thermal instability and even seizure of bearings. This is noted as the thermally induced preload problem, [Li 2004a], [Li 2004b], [Li 2003], [Lin 2003].

\subsection{Structure of proposed thermo-mechanical model}

A comprehensive method for simulations of thermo-mechanical issues in HS spindles has been proposed in [Holkup 2006]. The model uses axisymmetric 2D finite element method (FEM) for determination of thermal field and deformations. The thermal simulation is coupled with the mechanical simulation which continuously updates bearing condition and properties during transient solution.

There are three main steps of the iterative solution process:

- thermal analysis, with known thermal input data - computed by FEM;

- deformation analysis, with known thermal field and load - computed by FEM;

- internal condition of bearings, determined by system of algebraic equations according to [Jones 1960]. 


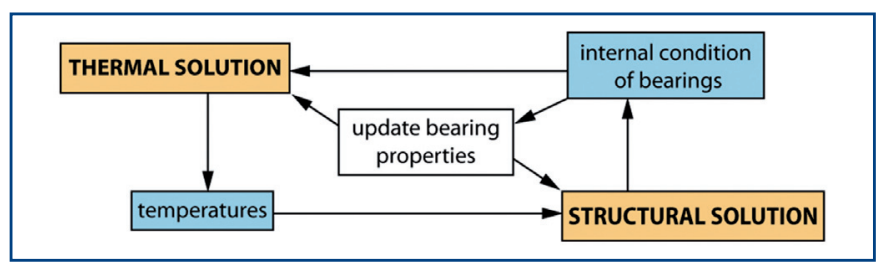

Figure 2. Solution flow diagram.

The closed-loop structure of the analysis was built using ANSYS FEM software with its APDL programmer's capabilities.

\subsection{Thermal model}

The model for simulation of spindle thermal field was build according to theories of heat transfer within solid materials and fluids, Figure 3.

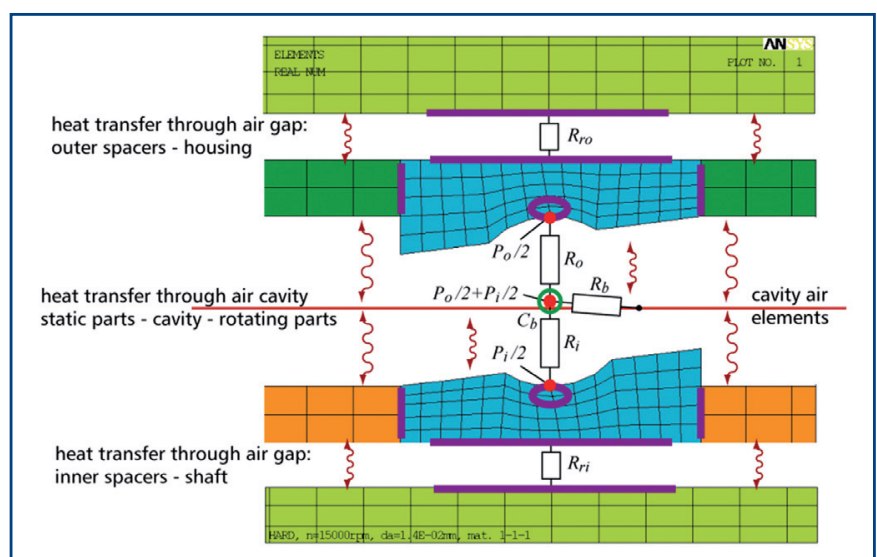

Figure 3. Heat transfer model of bearing surrounding.

Very important is the formula of heat generation in bearings - it depends on speed, preload, viscosity of lubricant, internal bearing design and other parameters. The theoretical formulas presented in [Harris 1991] were adjusted according to experimental results obtained on our test rig. These formulas match well in large range of operational conditions and bearing designs.

The character of the problem, performed simulations and experiments shows, that mainly the accuracy of the heat generation characteristic plays the key role in estimation of thermal issues in HS spindles.

\subsection{Structural mode}

The FEM analysis of spindle deformations considers the thermal deformations based on the current thermal field resulting from last thermal solution. The deformations caused by external and internal forces are considered as well.

\subsection{Bearing model}

In high speed operation of ball or roller bearings the rolling element centrifugal forces can be significantly large compared to the forces applied to the bearing externally. High speed affects the contact forces and angles, Figure 4, and also the dynamics of the bearing-supported rotor system.

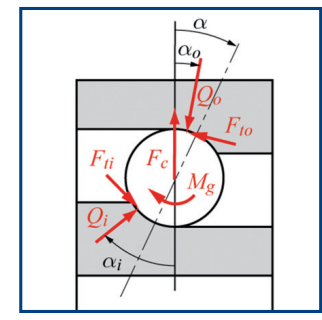

Figure 4. Ball loading
There are three kinds of equations that form the bearing model:

- Hertzian force-deflection characteristics of ball-ring contacts in normal direction;

- force and moment equilibrium on bearing balls, Figure 4;

- geometrical formulas stating the relation of bearing ring positions, contact angles and ball-ring normal deflection.

The bearing model from [Harris 1991] uses the Newton-Raphson method for computing the "internal condition" of bearings (contact forces, angles, rigidity) resulting from the "external condition" - ring position - calculated by FEM analysis. The result of the bearing model is always the complete stiffness matrix of each bearing in the assembly with diagonal as well as off-diagonal terms.

\subsection{Results and outputs of the thermo-mechanical model}

The results of the thermo-mechanical model are time-dependent and specific for each of the bearings:

- preloading force;

- stiffness matrix;

- relative radial and axial displacement between bearing rings.

Values of bearing ring's displacement and stiffness matrix are exported and used as input values for the subsequent mechanical spindle model.

\section{Model of spindle mechanics}

The model of spindle mechanics connecting finite-element and analytical description of the spindle components is used for modeling the spindle mechanics. The schema of the model is shown in Figure 5

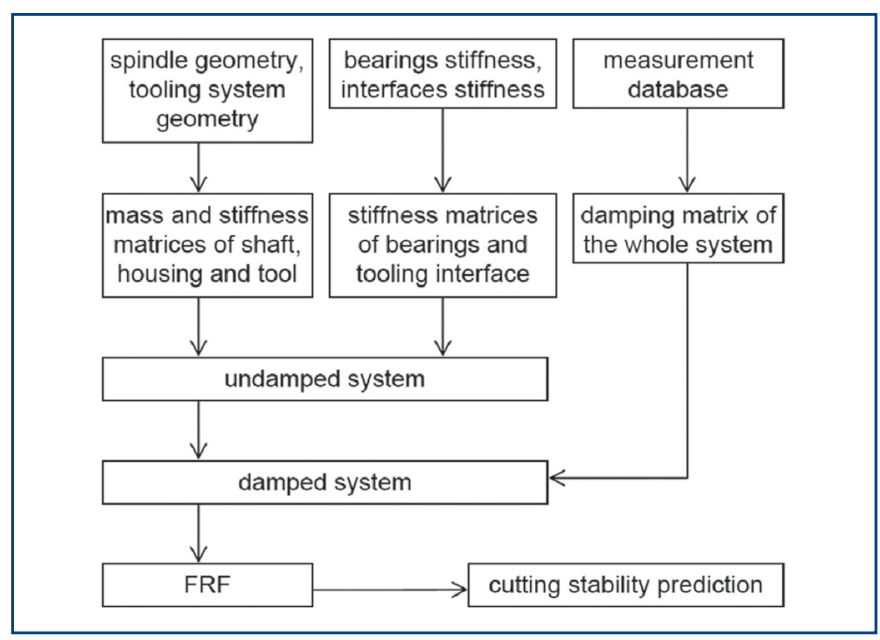

Figure 5. Schema of the model of spindle mechanics.

Mechanical properties of spindle shaft and housing are described by its stiffness and mass matrices. Properties of other spindle system components (bearings, tool holder - spindle interface) are described by corresponding stiffness matrices obtained separately. All matrices describe an undamped system which can be updated by additional damping information.

\subsection{Modeling of spindle geometry}

The hybrid model uses the FEM software Ansys for generation of mass and stiffness matrices of spindle shaft, spindle housing and the tool.

The full FEM model with 3D SOLID elements has too many degrees of freedom (DOF) therefore a reduction is necessary. The Guyan static reduction was used for decreasing the number of DOFs and only several nodes representing shaft/housing cross-sections were selected (see figure 6, reduction points are marked by arrows). The reduced mass and stiffness matrices of spindle shaft and housing 
were exported from Ansys to text files which were imported to Matlab and used for further analyses.

Example: The three-dimensional mapped mesh of spindle rotor has 9324 elements and 11736 nodes with 3 DOFs each. The reduced onedimensional model has 11 elements and 12 nodes with 6 DOFs each.

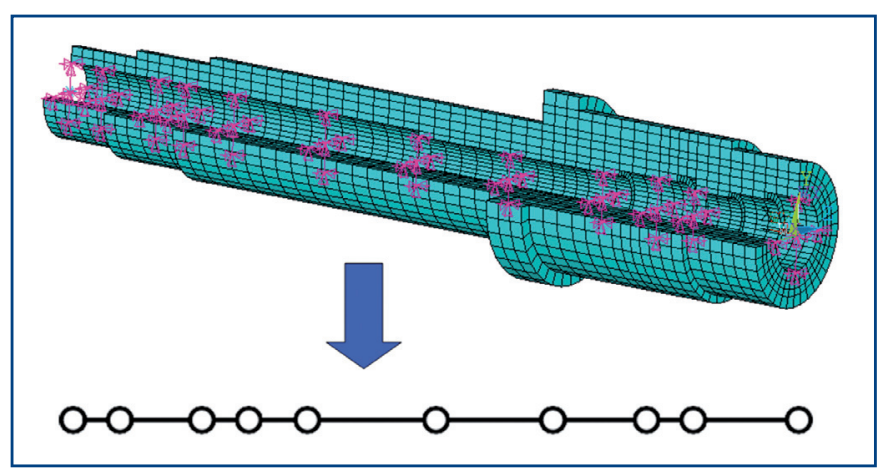

Figure 6. Meshed spindle shaft before reduction and equivalent reduced model.

A free-body modal analysis of spindle shaft was used for verification of the reduction method. The computations of modal properties of the three-dimensional model in Ansys and the reduced model in Matlab were compared with real measurement. The absolute total errors of both methods in comparison with the experiment are given in table 1. The non-reduced model in Ansys has an error about $1 \%$ and the reduced model in Matlab has an error about $2 \%$. We can see that the reduction of the part's geometry brings very small error into the model of it's dynamics in a free-free condition.

\begin{tabular}{|c|c|c|c|}
\hline \multicolumn{4}{|c|}{ Table 1. Errors of computational methods in comparison } \\
with experiment.
\end{tabular}

\subsection{Damping of the system}

Correct damping ratios can not be simulated and have to be obtained from measurements of a particular spindle. This is not useful for complex virtual design and optimization of cutting conditions [Altintas 2005]. It can be seen from statistical evaluation of measurements on real spindles (about 20 different types) that the most flexible frequencies have similar damping ratios. The most flexible frequency has mean damping of $3,2 \%$ and the second most flexible frequency has mean damping of $4,3 \%$. Although the variability of damping ratios is relatively large these values are usable for pilot computations.

\subsection{Structure of the hybrid model}

The process of building the hybrid model consists of several steps: The first step is to build the FEM 3D model of spindle shaft and housing and the tooling system. The FEM model is then reduced and stiffness and mass matrices are exported from Ansys into Matlab.

The second step is to prepare the stiffness matrices of bearings and interfaces. Bearing stiffness matrix is created by the bearing model which assumes the results of the thermo-mechanical analysis of the spindle. The interface stiffness is chosen according to the type of interface (HSK, ISO) and interface size. These stiffness matrices connect previous individual parts of the model together. The global stiffness and mass matrices are built and prepared for the next step.
The third step is to solve eigenvalue problem of undamped system. Results from this solution are eigen frequencies and eigen forms of spindle system. These are basis for next step.

The last step is a conversion of the model into the state space. The state space model can be used for various types of analyses e.g. dynamical response of the spindle to cutting forces.

\section{Example analysis}

\subsection{Spindle parameters}

The described thermo-mechanical-dynamical method was used for analyzing a particular grinding spindle. Main technical parameters of the spindle are:

- max. revolutions: $6000 \mathrm{rpm}$

- front bearing group: 4x FAG HCB7022C

- grinding wheel weight: $138 \mathrm{~kg}$

The geometry of the spindle shaft used for the computation is shown in Figure 7 before reduction. The grinding wheel was modeled using lumped mass elements at appropriate geometrical positions.

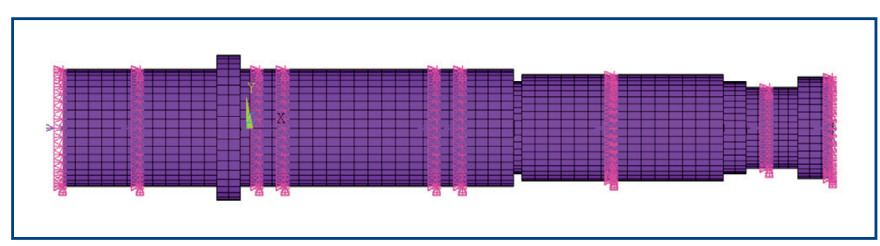

Figure 7. Spindle geometry before reduction.

\subsection{Thermo-mechanical model of the spindle}

A thermo-mechanical model of the front bearing assembly was built. The four bearings (sketch in Figure 8) are preloaded in two tandems in "O" configuration using rigid spacers. This kind of preload is very sensitive to thermal expansion of spindle parts; therefore the thermo-mechanical model must consider all heat sources and sinks accurately.

Several boundary conditions were introduced in the FEM model:

- heat source in each bearing as a function of operational parameters;

- heat source from rotor and stator of the electric motor;

- heat convection inside the spindle cavity between rotating and stationary parts;

- heat sink at the spindle housing representing the heat convection and conduction to the air and mating parts;

- heat sink at the grinding wheel.

\subsection{Results of the thermo-mechanical model}

The following Figures 8-10 show examples of results of the complex transient simulation. The resultant preload and stiffness characteristics are unique for each bearing, the numbers in the legend of Figures 9 and 10 refer to bearings from Figure 8 numbered from the left.

Results in Figure 9 show increasing axial preloading force due to thermal expansion during rotation. This phenomenon influences the bearing stiffness, Figure 10. Comparison of results of the thermomechanical model (i.e. model which updates its input data according to current thermal situation) and mechanical model (i.e. model without update) is shown in Figure 11.

The blue line shows the bearing radial stiffness as a function of increasing rotating speed due to centrifugal forces in one of the bearings. No thermal effects are considered. The stiffness at $6000 \mathrm{rpm}$ is about $40 \%$ smaller than at zero speed (mounting condition) due to centrifugal effects inside the bearing.

The colored squares show the stiffness of the first bearing of the spindle assembly at $6000 \mathrm{rpm}$ computed by the thermo-mechanical model. We can see that the stiffness at the beginning of the simulation is the same as the stiffness from the mechanical model. The thermal condition inside the spindle changes over time and the incre- 


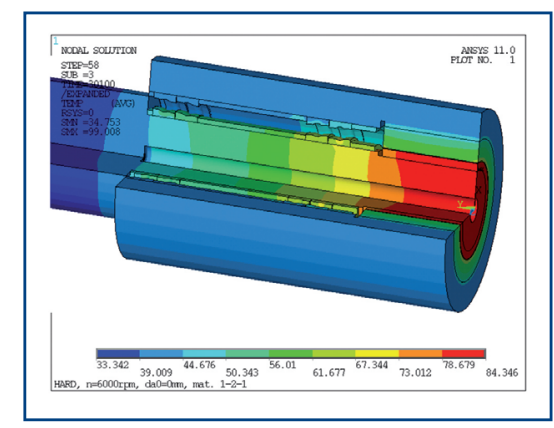

Figure 8. Schematic chart of the front part of the spindle - temperature field in the spindle.

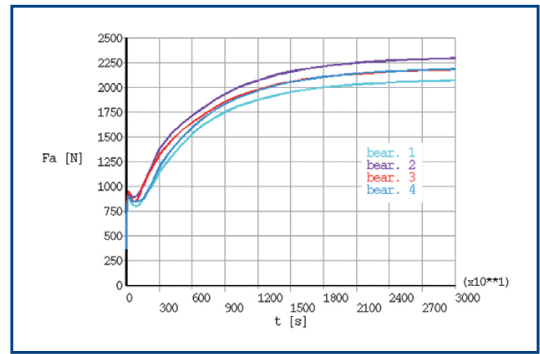

Figure 9. Preload force of all bearings.

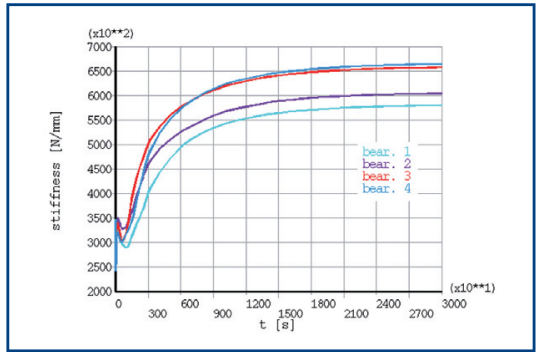

Figure 10. Radial stiffness of all bearings.

asing preload forces cause the increase of bearing stiffness. It can be seen, that the stiffness of basic mechanical model and the stiffness resulting from the thermo-mechanical simulation differs nearly $+300 \%$ after an eight-hour run.

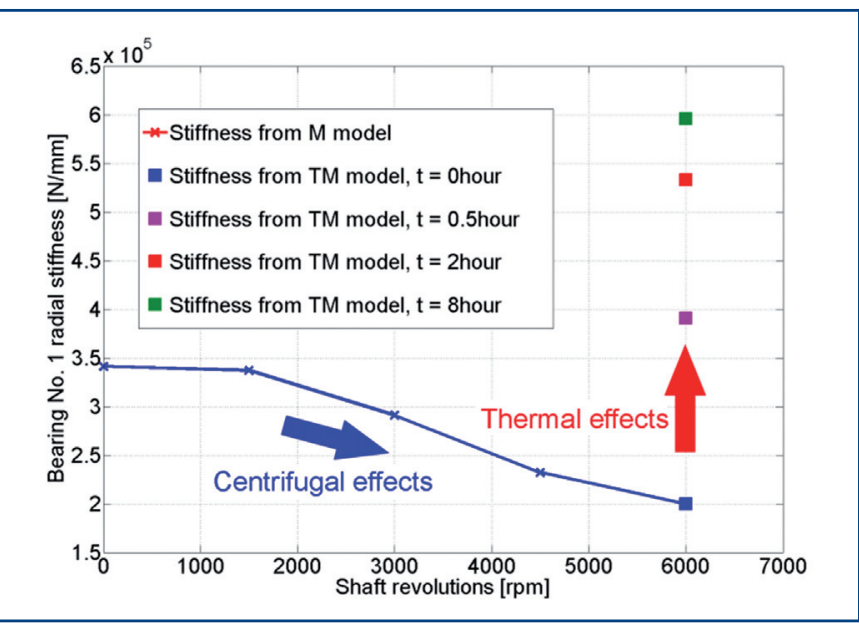

Figure 11. Influence of centrifugal and thermal effects on bearing stiffness.

\subsection{Results of the model of spindle dynamics}

The results of the thermo-mechanical model were used for computation of spindle dynamic properties; resultant eigenfrequencies are show in Table 2 and Figure 12.

\begin{tabular}{|c|c|c|c|c|}
\hline \multicolumn{5}{|c|}{$\begin{array}{c}\text { Table 2. Spindle eigenvalues changing } \\
\text { with the thermo-mechanical condition of bearings. }\end{array}$} \\
\hline \multirow{2}{*}{$\begin{array}{c}\text { Bending } \\
\text { frequency: }\end{array}$} & \multicolumn{4}{|c|}{ Spindle running time at $6000 \mathrm{rpm}$} \\
\hline & 0 hour & $\mathbf{0 , 5}$ hour & 2 hours & 8 hours \\
\hline 1. freq. & $153 \mathrm{~Hz}$ & $194 \mathrm{~Hz}$ & $212 \mathrm{~Hz}$ & $219 \mathrm{~Hz}$ \\
\hline 2. freq. & $313 \mathrm{~Hz}$ & $346 \mathrm{~Hz}$ & $353 \mathrm{~Hz}$ & $356 \mathrm{~Hz}$ \\
\hline 3. freq. & $401 \mathrm{~Hz}$ & $473 \mathrm{~Hz}$ & $500 \mathrm{~Hz}$ & $511 \mathrm{~Hz}$ \\
\hline 4. freq. & $933 \mathrm{~Hz}$ & $1088 \mathrm{~Hz}$ & $1157 \mathrm{~Hz}$ & $1185 \mathrm{~Hz}$ \\
\hline
\end{tabular}

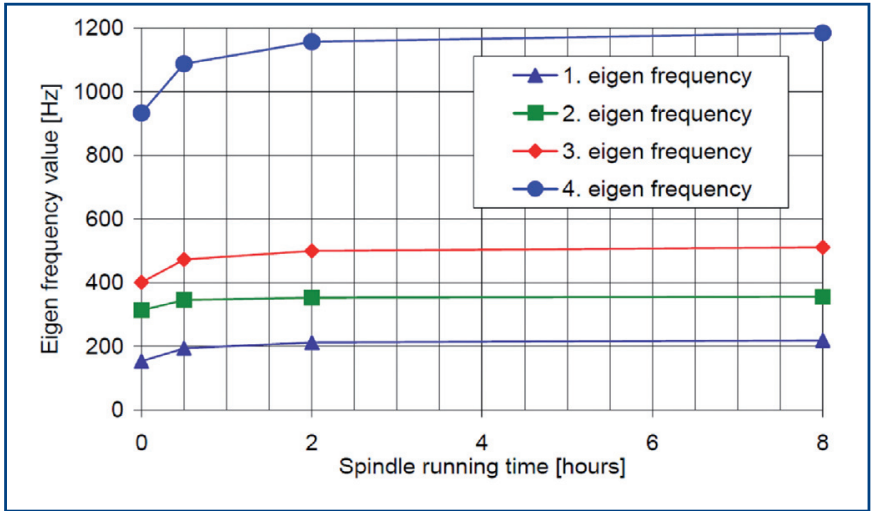

Figure 12. Spindle eigenvalues changing with the thermo-mechanical condition of bearings.

\section{Conclusions}

The results show that the thermal field that develops during spindle operation has significant effect on condition of spindle bearings, their properties and resultant spindle dynamics. The effect is very important mainly when position preload of bearings (rigid spacers) is used.

The proposed method of building the thermo-mechanical-dynamical models enables to predict spindle properties and to optimize their structure at the design stage. The main task for the future is to connect the mentioned sequential models into one user-friendly tool and to provide complex experimental verification in large range of spindle designs and operational conditions.

\section{Acknowledgement}

This research has been supported by the $1 \mathrm{M} 0507$ grant of the Ministry of Education of the Czech Republic and by the SKF company.

\section{References}

[Altintas 2005] Altintas, Y., Cao, Y., Virtual design and optimization of machine tool spindles. In: Annals of the CIRP. 2005, vol. 54, p. 379-382.

[Cao 2004] Cao, Y., Altintas, Y., A general method for the modeling of spindle-bearing system. Journal of Mechanical Desin. ASME 2004, vol. 126, p. 1089-1104, ISSN: 1050-0472.

[Harris 1991] Harris, T. A., Rolling Bearings Analysis. Third Edition. New York: John Wiley \& Sons 1991.

[Holkup 2006] Holkup, T., Holý, S., Complex modelling of spindle rolling bearings. Journal of Machine Engineering - Eficiency Development of Manufacturing Machines, vol. 6, no.3, 2006, p. 48-61. ISSN 1895-7595..

[Jones 1960] Jones, A.B., A general theory for elastically constrained ball and radial roller bearings under arbitrary load and speed conditions. Journal of Basic Design, Transactions of the ASME. 1960, s. 309-320.

[Kolar 2007] Kolar, P., Holkup, T., Modeling of a Machine Tools Spindle using a Hybrid Model. In: Proceedings of 3rd International Conference - Virtual Design and Automation. Poznań: Faculty of Mechanical Engineering and Management, Politechnika Poznańska, 2007. CD-ROM, paper No. S05_06_01_kolar. 8p 
[Li 2003] Li, H., Shin, Y. C., Analysis of bearing configuration effects on high speed spindles using an integrated dynamic thermo-mechanical spindle model. International Journal of Machine Tools and Manufacture. 2003, vol. 44, p. 347-364.

[Lin 2003] LIN, C. W., et al, An integrated thermo-mechanical-dynamical model to characterize motorized machine tool spindles during very high speed rotation. International Journal of Machine Tools and Manufacture. 2003, vol. 43, p. 1035-1050.

[Li 2004a] Li, H., Shin, Y. C., Integrated dynamic thermo-mechanical modeling of high speed spindles, part 1: model development. Journal of Manufacturing Science and Engineering, Transactions of the ASME. 2004, vol. 126, p. 148-158.

[Li 2004b] Li, H., Shin, Y. C., Integrated dynamic thermo-mechanical modeling of high speed spindles, part 2: solution procedure and validation. Journal of Manufacturing Science and Engineering, Transactions of the ASME. 2004, vol. 126, p. 159-168.

[Rantatalo 2007] Rantalalo, M., Aidanpää, J. O., Göransson, B., Norman, P., Milling machine spindle analysis using FEM and non-contact spindle excitation and response measurement. International Journal of Machine Tools and Manufacture. 2007, vol. 47, p. 1034-1045.

\section{Contacts}

Dipl. Ing. Petr Kolar, PhD.

Research Center for Manufacturing Technology

Faculty of Mechanical Engineering, CTU in Prague

Horska 3, 12800 Praha 2, Czech Republic

tel.: +420 221990 926, fax: +420221990999

e-mail: p.kolar@rcmt.cvut.cz 